Claudia Treder-Conrad ${ }^{1}$ Birgit Kloker $^{2}$

A. Dörschner ${ }^{1}$ F. Stäb ${ }^{1}$ F. Rippke ${ }^{1}$

\section{Wirksamkeit des antioxidativen Lichtschutzes bei polymorpher Lichtdermatose und Mallorca-Akne}

Ergebnisse einer multizentrischen Anwendungsbeobachtung

bei 577 Patienten

\author{
Efficacy of Antioxidant Photoprotection in Polymorphous Light Eruption \\ and Acne Aestivalis - Results of a Multicentric In-use-study in 577 Patients
}

\section{Zusammenfassung}

Die polymorphe Lichtdermatose (PLD) ist mit einer Prävalenz von 10 bis $20 \%$ die häufigste Fotodermatose und betrifft mehr als $90 \%$ aller Patienten mit lichtinduzierten Hautveränderungen. In der Pathogenese der PLD spielen Freie Radikale, entstanden unter dem Einfluss von UV-Strahlen, wahrscheinlich eine wesentliche Rolle. Die prophylaktische Wirksamkeit extern applizierter Antioxidantien wie alpha-Glucosylrutin und Tocopherolacetat bei experimentell fotoprovozierter PLD wurde bereits in verschiedenen Studien gezeigt. Im Rahmen einer Anwendungsbeobachtung an 577 Patienten wurden diese Ergebnisse unter Urlaubsbedingungen überprüft. Sowohl die klinische Hautsymptomatik der PLD bzw. Mallorca-Akne als auch der Juckreiz zeigten signifikante Verbesserungen im Vergleich des aktuellen zum letzten Urlaub. In nur 10,4\% der Fälle wurde ein Auftreten der PLD trotz Anwendung der Prüfprodukte dokumentiert. Die Aufenthaltsorte während des aktuellen Urlaubes zeigten dabei keine signifikanten Unterschiede zum letzten Urlaub. Die verwendeten Produkte erwiesen sich als sehr gut hautverträglich. Trotz des mit 39,2\% hohen Anteils an Patienten mit weiteren dermatologischen Vorerkrankungen, insbesondere einem atopischen Ekzem, wurden nur in 9 Fällen (1,5\%) Unverträglichkeitsreaktionen dokumentiert. Entsprechend positiv fielen die Bewertungen der Wirksamkeit und Hautverträglichkeit durch die Ärzte und Patienten aus.

\section{Abstract}

Polymorphous light eruption (PLE) is the most common photodermatosis with a prevalence of 10 to $20 \% .90 \%$ of patients with light-induced dermatoses are affected by PLE. In the pathogenesis of PLE free radicals, induced by UV-rays, seem to play an important role. The prophylactic efficacy of topically applied antioxidants, like alpha-Glucosylrutin and Tocepherolacetate, in experimentally provoked polymorphous light eruption has been shown in several clinical studies. These results were verified in a clinical-in-use study in 577 outpatients under field conditions during a summer holiday period. Clinical symptoms of PLE and acne aestivalis, as well as pruritus, showed significant improvements in comparison with last year's holiday. Only in $10,4 \%$ of cases PLE symptoms were documented although the patients had applied the test products. The holiday sites were comparable in both holidays and showed no significant difference. The application of the test products revealed a very good skin compatibility. Although there was a high portion of patients $(39,9 \%)$ suffering from additional dermatological diseases, especially atopic eczema, only 9 patients showed intolerance reactions to the test products. Accordingly, patients' and physicians' estimations on the efficacy and compatibility of the products were similarly positive. 
Die polymorphe Lichtdermatose stellt mit einer Prävalenz von $10-20 \%$ in Westeuropa und den USA eine der häufigsten Lichtdermatosen dar [1-3]. Bevorzugt betroffen sind Personen mit heller, lichtempfindlicher Haut und bis zu 80\% Frauen. Typische Prädilektionsstellen sind die Oberarme und Dekolletee, aber auch Gesicht, Beine und Hände können betroffen sein. Das klinische Bild unterscheidet sich interindividuell im papulären, papulovesikulären oder Plaque-Typ. Beim einzelnen Patienten zeigt sich meist dasselbe klinische Bild. Die Symptome treten in der Regel im Frühjahr nach der ersten intensiven Sonnenexposition auf. Das Aktionsspektrum liegt vorwiegend im UVA- (ca. 80\%), bei einem Teil der Patienten auch im UVB-Bereich (ca. 12\%), bzw. im UVA- und UVB-Bereich (ca. 8\%) [4].

Eine Immunreaktion vom verzögerten Typ, die durch UV-Strahlung ausgelöst und durch immunregulatorische Mechanismen aufrechterhalten wird, scheint als pathogenetische Ursache wahrscheinlich. Obwohl die genaue Pathogenese noch nicht geklärt ist, spielen Freie Radikale, entstanden unter UV-Einwirkung, vermutlich eine wichtige Rolle [5].

Die von Hjorth et al. 1972 [6] erstmals beschriebene MallorcaAkne stellt nach Tronnier und Heinrich [7,8] ein gegenüber der PLD eigenständiges, durch Peroxide aus Körperpflege- oder Sonnenschutzmitteln verursachtes Krankheitsbild dar [8]. Vorzugsweise tritt sie an talgdrüsenreichen Hautarealen wie Oberarmen, Schulterbereich, Rücken und Dekolletee auf [8-11]. In ihrem Bild ähnelt sie mit monomorphen, follikulär gebundenen Papeln einer Steroid-Akne, Komedonen und Pusteln fehlen meist [12]. Eine eindeutige ätiologische Zuordnung der Mallorca-Akne wird jedoch weiterhin kontrovers diskutiert: Nach Orfanos und Garbe [9] ist die Mallorca-Akne eine akneiforme Dermatose bei zu follikulärer Reaktionen disponierten Personen und entspricht in vieler Hinsicht einer polymorphen Lichtdermatose. Auch Altmeyer und Bacharach-Buhles [12] definieren die Mallorca-Akne als eine akneiforme Variante der PLD. Diese akneiformen Eruptionen können nach Hjorth et al. [6] und Braun-Falco et al. [11] unter UV-Exposition auch ohne Lipide und Emulgatoren aus Lichtschutzmitteln oder anderen Kosmetika entstehen. Dennoch wird allgemein die Vermeidung öliger, zu lipophiler Lichtschutzmittel zugunsten von Cremes und insbesondere Gelen empfohlen $[7-9,12]$.

Mit alpha-Glucosylrutin (AGR) steht inzwischen ein Antioxidans pflanzlicher Herkunft zur Verfügung, dessen ausgeprägte Wirksamkeit auf verschiedene Parameter oxidativer Zellschädigungen in-vitro und in-vivo nachgewiesen werden konnte $[13,14]$. In mehreren klinischen und experimentellen Studien konnte auch eine prophylaktische Wirksamkeit dieses topisch in Kombination mit Tocopherolacetat applizierten Flavonoids auf die polymorphe Lichtdermatose gezeigt werden [15-17]. Darüber hinaus gab es auch erste Hinweise auf eine Wirksamkeit bei Mallorca-Akne [16]. Im Rahmen einer breit angelegten Anwendungsbeobachtung, die bei 577 Patienten durchgeführt wurde, sollten diese Ergebnisse unter Urlaubsbedingungen sowohl bei Patienten mit anamnestischer PLD als auch Mallorca-Akne überprüft werden.
In die Anwendungsbeobachtung wurden insgesamt 577 Patienten mit anamnestisch bekannter PLD und/oder Mallorca-Akne, die aktuell jedoch erscheinungsfrei waren, aus 108 dermatologischen Praxen einbezogen.

Zu Beginn der Untersuchung erfolgte eine Anamneseerhebung zum Alter, Geschlecht und Art der PLD (papulöser, papulovesikulöser oder Plaque-artiger Typ). Die Art der Diagnosestellung der PLD oder Mallorca-Akne (klinisch/anamnestisch oder durch Fotoprovokation) wurde dokumentiert. Ebenso wurden der Schweregrad (Abb.1 u.2) und der Hauptlokalisationsort der PLD bzw. Mallorca-Akne dokumentiert, wobei die bereits in früheren $\mathrm{Ar}$ beiten verwendeten diagnostischen Kriterien Anwendung fanden (Tab. 1 u. 2) [15,16].

Der Hauttyp wurde nach Fitzpatrick bestimmt. Weitere dermatologische Vorerkrankungen, Allergien sowie medikamentöse Therapien und eventuelle Vorbehandlungen wurden dokumentiert. Ebenso erfolgten Angaben zum letzten Urlaub, wie Aufenthaltsort, Symptome und Behandlung der PLD bzw. MallorcaAkne im letzten Urlaub.

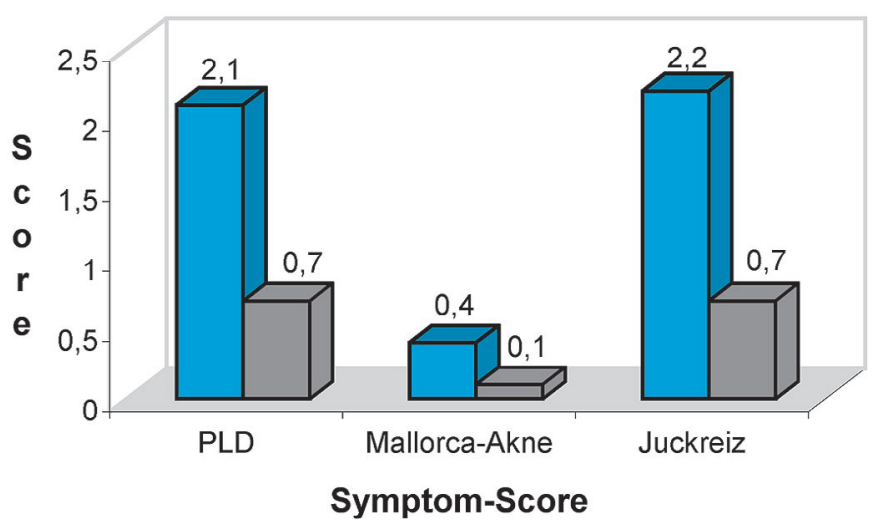

$\square$ letzter Urlaub $\square$ aktueller Urlaub

Abb. 1 Ausprägung der Hautsymptomatik im Prüfareal im aktuellen im Vergleich zum letzten Urlaub.

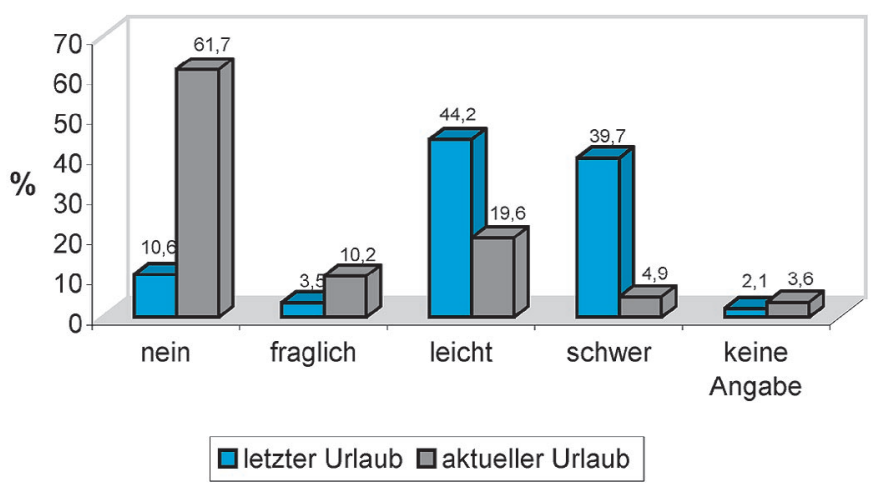

Abb. 2 Schweregrad des Juckreizes im Prüfareal im aktuellen im Vergleich zum letzten Urlaub. 
Tab. 1 Einteilung des Schweregrades der Mallorca-Akne

\begin{tabular}{|c|c|c|}
\hline Grad 0 & $\begin{array}{l}\text { Pigmentierung, flächige Rötung, } \\
\text { keine follikulär gebundene Papeln }\end{array}$ & $\begin{array}{l}\text { keine klinisch manifeste } \\
\text { Mallorca-Akne }\end{array}$ \\
\hline Grad I & $\begin{array}{l}\text { Juckreiz und wenige kleine follikuläre } \\
\text { Papeln }(<20) \text { im Bereich Gesicht, } \\
\text { Schultern, Streckseiten der Oberarme, } \\
\text { Brust, Rücken }\end{array}$ & fragliche Mallorca-Akne \\
\hline Grad II & $\begin{array}{l}\text { Juckreiz und mäßig viele Papeln } \\
(20 \text { - 100) im Bereich Gesicht, } \\
\text { Schultern, Streckseiten der } \\
\text { Oberarme, Brust, Rücken }\end{array}$ & $\begin{array}{l}\text { klinisch manifeste } \\
\text { Mallorca-Akne } \\
\text { (leichte Form) }\end{array}$ \\
\hline Grad III & $\begin{array}{l}\text { Zahlreiche follikuläre Papeln (> 100) } \\
\text { im Bereich Gesicht, Schultern, Streck- } \\
\text { seiten der Oberarme, Brust, Rücken }\end{array}$ & $\begin{array}{l}\text { klinisch manifeste } \\
\text { Mallorca-Akne } \\
\text { (schwere Form) }\end{array}$ \\
\hline
\end{tabular}

Tab. 2 Einteilung des Schweregrades der PLD

\begin{tabular}{|c|c|c|}
\hline Grad 0 & $\begin{array}{l}\text { Pigmentierung, flächige Rötung, } \\
\text { keine Papulovesikeln oder Plaques }\end{array}$ & $\begin{array}{l}\text { keine klinisch manifeste } \\
\text { PLD }\end{array}$ \\
\hline Grad I & $\begin{array}{l}\text { fleckiges Erythem } \\
\text { und } \\
\text { Juckreiz }\end{array}$ & fragliche PLD \\
\hline Grad II & $\begin{array}{l}\text { Papulovesikeln/Papeln }=20 \text { Stück } \\
\text { im Provokationsfeld }(5 \times 5 \mathrm{~cm}) \\
\text { oder } \\
\text { Plaque }=50 \% \text { des Provokationsfeldes } \\
(5 \times 5 \mathrm{~cm})\end{array}$ & $\begin{array}{l}\text { klinisch manifeste PLD } \\
\text { (leichte Form) }\end{array}$ \\
\hline Grad III & $\begin{array}{l}\text { Papulovesikeln/Papeln }>20 \text { Stück im } \\
\text { Provokationsfeld }(5 \times 5 \mathrm{~cm}) \\
\text { oder } \\
\text { Plaque }>50 \% \text { des Provokationsfeldes } \\
(5 \times 5 \mathrm{~cm}) \\
\text { oder } \\
\text { Vorkommen von Bullae oder } \\
\text { Hämorrhagien }\end{array}$ & $\begin{array}{l}\text { klinisch manifeste PLD } \\
\text { (schwere Form) }\end{array}$ \\
\hline
\end{tabular}

$483(83,7 \%)$ der teilnehmenden Patienten waren weiblichen und 92 (15,9\%) männlichen Geschlechts, bei 2 Patienten fehlten hierzu die Angaben.

Das durchschnittliche Alter der Patienten betrug 37,8 $( \pm 14,1)$ Jahre, wobei der größte Anteil (30\%) zu der Gruppe der 30- bis 39-jährigen gehörte, 22,2\% gehörten zur Gruppe der 20- bis 29-jährigen und 19,8\% zur Gruppe der 40- bis 49-jährigen. 4 Patienten waren unter 10 Jahren und 12 Patienten über 70 Jahre alt.

Bei 359 (62,2\%) der Patienten wurde der Hauttyp II nach Fitzpatrick angegeben, bei 129 Patienten (22,4\%) Typ III und bei 6 Patienten (1,0\%) Typ IV. Bei $8(1,4 \%)$ Patienten fehlte die entsprechende Angabe.

39,2\% der Patienten gaben eine zusätzliche dermatologische Vorerkrankungen an, wobei hier das atopische Ekzem mit 18,2\% am häufigsten genannt wurde. Insgesamt lag bei $22,0 \%$ aller Patienten eine Sensibilisierung vor. 58,3\% dieser Patienten gaben eine Kontaktallergie an.

Bei allen Patienten bestand eine klinisch bzw. anamnestisch dokumentierte polymorphe Lichtdermatose (534/92,5\%) und/oder
Tab. 3 Besuchte Urlaubsländer in der letzten und aktuellen Saison

\begin{tabular}{|lrrrr|}
\hline Urlaubsland & \multicolumn{3}{c}{ letzter Urlaub } & \multicolumn{2}{c}{ aktueller Urlaub } \\
& $\boldsymbol{n}$ & $\mathbf{\%}$ & $\boldsymbol{n}$ & $\%$ \\
\hline Spanien & 152 & 26,3 & 169 & 29,8 \\
\hline Italien & 62 & 10,7 & 74 & 13,0 \\
\hline Griechenland & 47 & 8,1 & 31 & 5,5 \\
\hline Türkei & 44 & 7,6 & 38 & 6,7 \\
\hline Frankreich & 29 & 5,0 & 30 & 5,3 \\
\hline Österreich & 28 & 4,9 & 19 & 3,3 \\
\hline Deutschland & 79 & 13,7 & 75 & 13,2 \\
\hline
\end{tabular}

Tab. 4 Häufigste Aufenthaltsorte im letzten und aktuellen Urlaub

\begin{tabular}{|lrrrr|}
\hline häufigster Aufenthaltsort & \multicolumn{2}{c}{ letzter Urlaub } & \multicolumn{2}{c}{ aktueller Urlaub } \\
& $\boldsymbol{n}$ & $\%$ & $\boldsymbol{n}$ & $\%$ \\
\hline am Meer & 447 & 77,5 & 432 & 74,9 \\
\hline in den Bergen & 47 & 8,1 & 50 & 8,7 \\
\hline in der Stadt & 36 & 6,2 & 32 & 5,5 \\
\hline auf dem Land & 38 & 6,6 & 42 & 7,3 \\
keine Angabe & 9 & 1,6 & 21 & 3,6 \\
\hline total & 577 & 100,0 & 577 & 100,0 \\
\hline
\end{tabular}

eine Mallorca-Akne (70/12,1\%). Eine Doppelnennung war möglich.

Bei der PLD handelte es sich bei 48,7\% um einen papulösen Typ, bei 35,8\% um einen papulovesikulösen Typ und bei 9,9\% um einen Plaque-artigen Typ. Bei 5,6\% der Betroffenen fehlte hierzu eine Angabe. Als Hauptprädilektionsstelle der PLD bzw. Mallorca-Akne-Symptome wurde das Dekolletee mit 82,5\% angegeben, gefolgt von Oberarm (67,2\%) und Schulter (47,7\%), wobei Mehrfachnennungen möglich waren. Aufgrund der anamnestischen Angaben zur Hauptprädilektionstelle der PLD bzw. MallorcaAkne wurde das Prüfareal für den aktuellen Urlaub individuell festgelegt.

Die PLD- und Mallorca-Akne-Symptome waren anamnestisch bei ca. der Hälfte der Patienten nach mäßiger Bestrahlung aufgetreten, bei jeweils ca. einem Viertel der Patienten traten die Symptome nach geringer oder starker Belichtung auf.

Den letzten Urlaub hatte der überwiegende Anteil der Patienten (77,5\%) am Meer und in Südeuropa verbracht (Tab. 3u.4).

Vor dem aktuellen Urlaub wurde bei 82 (14,2\%) der Patienten eine Vorbehandlung durch „Hardening“ durchgeführt, im Vergleich zu 63 (10,9\%) im letzten Urlaub.

Die Patienten wurden angewiesen, 7 Tage vor Ihrem Urlaub Eucerin $^{\circledR}$ Gel-Creme Phase 1 (Hydrodispersionsgel mit 0,25\% AGR $+1 \%$ Tocopherolacetat) zweimal täglich auf den festgelegten Prüfarealen zu verwenden. Während des Urlaubes sollte Eu- 
cerin ${ }^{\circledR}$ Gel-Creme 15 Phase 2 (wie oben, zusätzlich mit UV-Filtern LSF 15) als Sonnenschutzprodukt verwendet werden, d.h. es erfolgte auch eine Anwendung außerhalb des Prüfareals.

Die Prüfprodukte setzten sich entsprechend der internationalen Nomenklatur für Kosmetische Inhaltstoffe (INCI) wie folgt zusammen:

Eucerin ${ }^{\circledR}$ Gel-Creme Phase 1: Aqua, Hydrogenated Coco-Glycerides, Glycerin, Dimethicone, Alcohol Denat., Paraffinum Liquidum, Cera Microcristallina, Octyldodecanol, C12 - 15 Alkyl Benzoate, Dicaprylyl Ether, Xanthan Gum, Tocopheryl Acetate, Acrylates/C10 - 30 Alkyl Acrylate Crosspolymer, Phenoxyethanol, Methylparaben, Glucosylrutin, Trisodium EDTA, Sodium Citrate, Citric Acid, PEG-4 Laurate, PEG-4 Dilaurate, Isoquercitrin, PEG-4, Iodopropynyl Butylcarbamate.

Eucerin $^{\circledR}$ Gel-Creme Phase 2 LSF 15: Aqua, Ethylhexyl Methoxycinnamate, Ethylhexyl Triazone, Glycerin, Butyl Methoxydibenzoylmethane, Butylene Glycol Dicaprylate/Dicaprate, C12 - 15 Alkyl Benzoate, Hydrogenated Coco-Glycerides, Dimethicone, Alcohol Denat., C18-36 Acid Triglyceride, Tocopheryl Acetate, Xanthan Gum, Cera Microcristallina, Phenoxyethanol, Acrylates/C10 - 30 Alkyl Acrylate Crosspolymer, Paraffinum Liquidum, Methylparaben, Glucosylrutin, Trisodium EDTA, Sodium Citrate, Citric Acid, PEG-4 Laurate, PEG-4 Dilaurate, Isoquercitrin, PEG-4, Iodopropynyl Butylcarbamate, BHT.

Eine medikamentöse systemische Prophylaxe galt als Ausschlusskriterium, ebenso die Verwendung von Präparaten an den Prüfarealen, die den antioxidativen Status beeinflussen könnten, wie z. B. Vitamine, Antiphlogistika oder Kortikosteoride.

Zum Abschluss wurde die Wirksamkeit der Prüfprodukte im Prüfareal durch den Arzt und den Patienten selbst dokumentiert (sehr gut, gut, mäßig, schlecht).

Kriterien zur Beurteilung der Wirksamkeit waren das Auftreten von Symptomen der PLD oder Mallorca-Akne (Entwicklung eines Erythems u./o. Bläschen, Papeln) und von Juckreiz, jeweils im Prüfareal. Diese wurden mit einer 4-stufigen Skala $(0=$ keine, 1 = fragliche, 2 = leichte, 3 = schwere Symptome/Juckreiz) durch den Arzt jeweils bei der Aufnahme- und Abschlussuntersuchung bewertet.

Die nach Studienabschluss eingegangenen Dokumentationsbogen wurden vor der Codierung einer ersten Plausibilitätskontrolle und, nach der doppelten Erfassung der Daten, einer weiteren Kontrolle unterzogen. Die Überprüfung, Korrektur und Auswertung der Rohdaten erfolgte mit SAS-Version 8.0 auf einem IBM-kompatiblen PC unter MS-Windows. Quantitative Merkmale wurden mit arithmetischem Mittel, Standardabweichung, Minimum, 1. Quartil, Median, 3. Quartil und Maximum beschrieben. Bei qualitativen Merkmalen wurden absolute und relative Häufigkeiten angegeben.
Ergebnisse

Von allen 577 Patienten lagen nach Untersuchungsabschluss auswertbare Prüfbogen vor.

Für 514 Patienten (89,1\%) konnte eine regelmäßige 7-tägige Vorbehandlung mit Eucerin ${ }^{\circledR}$ Gel-Creme Phase 1 im Prüfareal dokumentiert werden und 42 Patienten (7,3\%) führten eine teilweise Vorbehandlung durch. 529 Patienten (91,7\%) verwendeten Eucerin $^{\circledR}$ Gel-Creme Phase 2 regelmäßig als Sonnenschutzprodukt, speziell im Prüfareal, 27 Patienten (4,7\%) teilweise und bei 18 der Patienten fehlten hierzu die Angaben.

Beim Vergleich der Urlaubsorte vom letzten und aktuellen Urlaub konnte kein signifikanter Unterschied $(p=0,6)$ im t-Test für abhängige Stichproben ermittelt werden.

Auch im aktuellen Urlaub hielt sich der Großteil der Patienten am Meer auf $(74,9 \%)$ und wie im letzten Urlaub wurde Südeuropa bevorzugt (Tab. 3 u.4).

Bei der Ermittelung der Score-Werte und der Schweregradbeurteilung für die PLD-Symptome, Symptome der Mallorca-Akne sowie für den Juckreiz jeweils im Prüfareal ergaben sich im t-Test für abhängige Stichproben jeweils signifikante Verbesserungen (jeweils $\mathrm{p}<0,01$ ) im Vergleich des aktuellen zum letzten Urlaub. Die durchschnittlichen Score-Werte verringerten sich dabei von 2,1 auf 0,7 (PLD-Symptome), von 0,4 auf 0,1 (Symptome der Mallorca-Akne) bzw. von 2,2 auf 0,7 (Juckreiz).

Entsprechend verringerte sich der Anteil der unter leichtem oder schwerem Juckreiz leidenden Patienten von 83,9\% im letzten Urlaub auf 24,5\% im aktuellen Urlaub. 61,7\% waren im aktuellen Urlaub hinsichtlich des Juckreizes vollständig beschwerdefrei.

Im aktuellen Urlaub waren 54,9\% der Patienten bez. der PLDSymptome beschwerdefrei. Dies stellte im Vergleich zum letzten Urlaub, in dem nur 9,2\% der Patienten beschwerdefrei waren, eine deutliche Verbesserung dar. Im aktuellen Urlaub litten nur noch 6,4\% der Patienten unter schweren PLD-Symptomen, gegenüber 34,3\% im letzten Urlaub (Abb. 3).

Auch bei der Beurteilung der Mallorca-Akne zeigt sich eine signifikante $(p<0,01)$ Verbesserung hinsichtlich des Schweregrades (Abb. 4).

Nur bei 27 (4,7\%) der Patienten traten unerwünschte Effekte auf. Dies war bei 8 Patienten ein erneutes Auftreten der PLD-Symptome. 9 Patienten beklagten eine Gelbverfärbung der Kleidung und für 7 Patienten wurde eine Unverträglichkeitsreaktion auf eines der Prüfprodukte dokumentiert. In nur einem Fall kam es zum Auftreten eines Sonnenbrandes im Prüfareal. Bei 2 Patienten fehlten die Angaben zur Art der unerwünschten Effekte.

Insgesamt wurde bei 16 Patienten ein Studienabbruch dokumentiert, bei 8 Patienten aufgrund unerwünschter Effekte, 2 Patienten hatten ihren Urlaub nicht angetreten und 6 Patienten waren zur vereinbarten Abschlussuntersuchung nicht erschienen. 


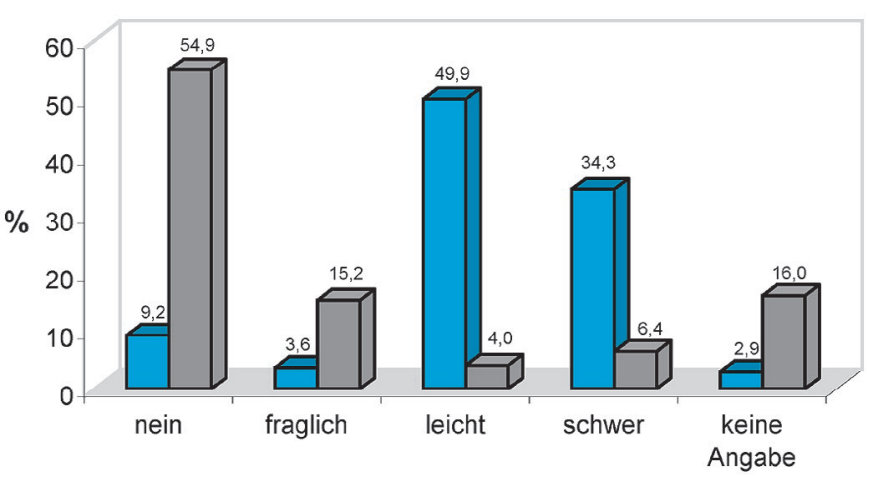

letzter Urlaub $\square$ aktueller Urlaub

Abb. 3 Schweregrad der PLD-Symptome im Prüfareal im aktuellen im Vergleich zum letzten Urlaub.

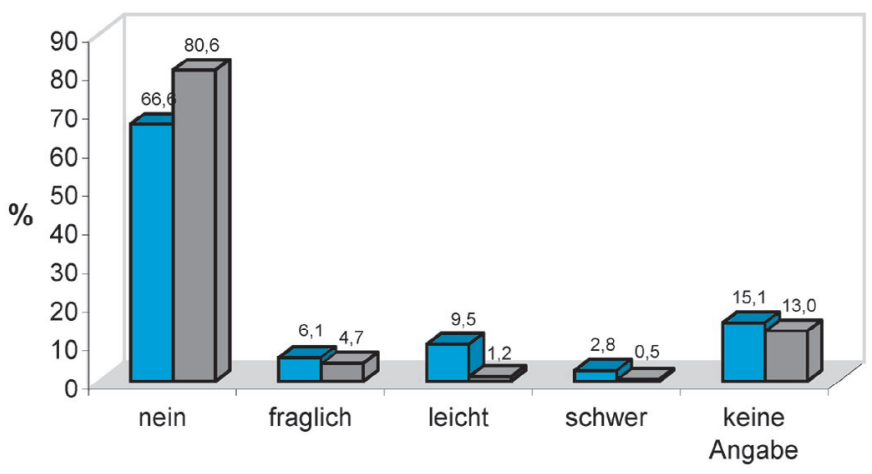

$\square$ letzter Urlaub $\square$ aktueller Urlaub

Abb. 4 Schweregrad der Mallorca-Akne im Prüfareal im aktuellen im Vergleich zum letzten Urlaub.

Die Gesamtbeurteilung der Wirksamkeit durch Arzt und Patient führte zu annähernd gleichen Ergebnissen. Die Ärzte beurteilten die Wirksamkeit in 81,9\% der Fälle als „sehr gut“ bis „gut“ und die Patienten in 81,8\%. Die Weiterempfehlung der Präparate befürworteten 76,3\% der Ärzte und 68,8\% der Patienten gaben an, sie weiter zu verwenden.

\section{Diskussion}

Die in vorangegangenen klinisch-experimentellen Untersuchungen [15 - 17] gezeigte prophylaktische Wirksamkeit extern applizierter Antioxidantien bei der polymorphen Lichtdermatose konnte in dieser großangelegten Anwendungsbeobachtung auch unter Praxisbedingungen bestätigt werden. Darüber hinaus konnte die Wirksamkeit auch bei der Mallorca-Akne bestätigt werden: Sowohl die klinischen Hautsymptome als auch der Juckreiz zeigten bei PLD und Mallorca-Akne signifikante Verbesserungen im Vergleich des aktuellen Urlaubes zum letzten Urlaub. Lediglich bei $10,4 \%$ der Fälle wurde trotz Verwendung der Prüfprodukte ein Auftreten der PLD dokumentiert.
Ziel der Prophylaxe ist es, bei den betroffenen Patienten die Symptome und den Juckreiz der PLD bzw. Mallorca- Akne während der „Sonnensaison“ möglichst zu verhindern oder zumindest deutlich zu reduzieren. Als Prophylaxe-Maßnahmen sind unterschiedliche Möglichkeiten beschrieben worden. Hierzu zählen zunächst allgemeine Lichtschutzmaßnahmen wie das Tragen lichtundurchlässiger Kleidung, Meidung intensiver UVBestrahlung und Verwendung von Lichtschutzmitteln mit Breitbandfiltern, die Schutz im UVB- und insbesondere auch im UVABereich bieten [4]. Als weitere prophylaktische Maßnahme wird ein systemischer UV-Schutz mit Betacarotin oder Nikotinsäureamid diskutiert, wobei ein Lichtschutzfaktor von ca. 2 erzielt werden kann. Eine systemische immunsuppressive Therapie mit Glukokortikoiden ist wirksam, sollte jedoch aufgrund der möglichen Nebenwirkungen schweren Fällen der PLD vorbehalten bleiben. Weitere Optionen sind die UV-Therapie oder auch die PUVA-Therapie [18].

Wie in dieser Studie gezeigt werden konnte, ist die lokale Anwendung der Kombination des hochwirksamen Antioxidans alpha-Glucosylrutin mit Tocopherolacetat eine wirksame Möglichkeit zur Prophylaxe der polymorphen Lichtdermatose und auch der Mallorca-Akne.

Trotz des Vorliegens weiterer dermatologischer Vorerkrankungen, insbesondere eines atopischen Ekzems bei 39,2\% und einer bekannten Kontaktallergie bei 22,0\% der Patienten, wurden nur in 7 Fällen Unverträglichkeitsreaktionen auf die Prüfprodukte dokumentiert. Damit konnte die gute Verträglichkeit der Prüfprodukte, auch bei Patienten mit Disposition zu irritativen oder allergischen Hautreaktionen, ebenfalls bestätigt werden. Die prophylaktische Anwendung extern applizierter Antioxidantien wie AGR und Tocopherolacetat stellt damit eine sinnvolle Ergänzung des Spektrums der Präventionsmöglichkeiten bei Patienten mit polymorpher Lichtdermatose und Mallorca-Akne dar.

\section{Literatur}

${ }^{1}$ Berg M. Epidemiological studies of the influence of sunlight on the skin. Photodermatology 1989; 6: 80-84

${ }^{2}$ Ros A-M. Current aspects of polymorphous light eruptions in Sweden. Photodermatology 1986; 3: 298-302

${ }^{3}$ Morison WL, Stern RS. Polymorphous light eruption: a common reaction uncommonly recognized. Acta Derm Venereol 1982; 62: 409-413

${ }^{4}$ Lehmann P. Polymorphe Lichtdermatose: Was hilft wirklich? In: Plewig G, Degitz K (Hrsg). Fortschritte der praktischen Dermatologie und Venerologie 2000. Berlin, Heidelberg: Springer, 2000

${ }^{5}$ Hölzle E. Ein wirksamer UV-A-Schutz ist die Basis der Prävention. Dt Derm 2000; 6: 409-413

${ }^{6}$ Hjorth N, Sjolin K, Sylvest B, Thomsen K. Acne aestivalis - Mallorca acne. Acta Dem-Venerol 1972; 52: 61 - 63

${ }^{7}$ Tronnier H, Heinrich U. Die Mallorca-Akne (follikulär-papulöse Lichtdermatose) als eigenes Krankheitsbild - aktueller Wissensstand. SÖFW-Journal 2000; 126: 54-55

8 Tronnier H, Heinrich U. Die polymorphen Lichtdermatosen. Studie zur Pathogenese, Prophylaxe und Therapie. Akt Dermatol 1994; 20: 220-226

${ }^{9}$ Orfanos C, Garbe C. Therapie der Hautkrankheiten. 2. Aufl. Berlin, Heidelberg: Springer, 2002

${ }^{10}$ Plewig G, Kligman A. Akne und Rosazea. 2. Aufl. Berlin, Heidelberg: Springer, 1994

${ }^{11}$ Braun-Falco O, Plewig G, Wolff HH. Dermatologie und Venerologie. 4. Aufl. Berlin, Heidelberg: Springer, 1996 
${ }^{12}$ Altmeyer P, Bacharas-Buhles M. Springer-Enzyklopädie Dermatologie, Allergologie, Umweltmedizin. Berlin, Heidelberg, New York: Springer, 2002

${ }^{13}$ Stäb F, Lanzendörfer G, Schönrock U, Wenck H. Novel antioxidants: new strategies in product stabilization and skin protection. SÖFW Journal 1998; 10: 604-613

${ }^{14}$ Stäb F, Wolber R, Mundt C, Blatt T, Will K, Keyhani R, Rippke F, Max H, Schönrock U, Wenck H, Moll I, Hölzle E, Wittern K. Alpha-Glucosylrutin - an innovative antioxidant in skin protection. SÖFW Journal 2001; 9: $2-8$

${ }^{15}$ Hadshiew I, Stäb F, Untidt S, Bohnsack K, Rippke F, Hölzle E. Effects of topically applied antioxidants in experimentally provoked polymorphous light eruption. Dermatology 1997; 195: 362 - 368
${ }^{16}$ Rippke F, Wendt G, Bohnsack K, Dörschner A, Stäb F, Hölzle E, Moll I. Results of photoprovocation and field studies on the efficacy of a novel topically applied antioxidant in polymorphous light eruption. J Derm Treat 2001; 12:3-8

${ }^{17}$ Kowalzick L, Suckow M, Carl A, Waldmann T, Bohnsack K, Pönnighaus J. UV-B induzierte polymorphe Lichtdermatose: Protektion durch topische Antioxidantien. Akt Dermatol 1998; 24: $11-14$

${ }^{18}$ Kowalzick L, Mensing H, Wagner G. Polymorphe Lichtdermatose. In: Kowalzick L (Hrsg). Praxis der Lichtdermatosen. 1. Aufl. Bremen: UniMed, 2000 